\title{
Issues with using Landsat based Surface Temperature for Investigating Linkage between Local Climate Zones and Surface Urban Heat Island in Different Seasons: A Case Study of Manhattan, N.Y.
}

\author{
Parth Bansal \\ Graduate School of Environmental Studies, Seoul National University
}

\begin{abstract}
This study was conceptualized to investigate differences in surface temperature profile of Local Climate Zones (LCZ) classes in different seasonal conditions. Manhattan was selected as case study due to its dense, but heterogeneous built-up profile and presence of green area which formed the baseline for temperature comparison. However, this study failed to find significant results, in terms of the distinct Urban Heat Island (UHI) feature often reported in literature. Instead, this study suggests that in the case of Manhattan UHI is predominantly within $\pm 0.5 \mathrm{C}^{\circ}$ except during summer season. In summer season, where more difference in built and green LCZ is observed, the noise in data, defined by standard deviation of surface temperature in the class, is also higher. Thus, our study concludes that Landsat based surface temperature should be used with extreme caution to investigate UHI since most imagery is taken during day time.
\end{abstract}

\section{Introduction}

The impact of urbanization on the environment has continued to receive attention in climate studies to inform decision making in urban planning. The relative difference in urban area's temperature, as compared to its rural surround, termed as Urban Heat Island (UHI), has been documented in literature under different urban and weather context. With climate change projected to further exaggerate UHI related issues (Estrada et al., 2017), UHI has recently also attracted attention of decision makers who aims to tackle increase incidences and intensity of heat waves.

However, much of the literature on UHI has been fragmented by different methodologies, making any sort of meta-analysis difficult. Generally, the literature has defined UHI as a simple difference in temperature between urban and rural area. However, since urban \& rural are subjective terminologies, the rural area of a given study might have been considered urban in another. Besides, urban form itself is diverse, and this difference is expected to produce a different UHI feature. This issue has been well explored by Oke (2006).

In response, Local Climate Zones (LCZ) framework has been proposed by Stewart and Oke (2012), consisting of 10 built-up and 7 land cover classes, each defined by 7 parameters. The stated aim of the framework is to develop a classification which improves comparison across study areas and make reporting of UHI magnitude objective. The latter aspect has received considerable attention, with a number of studies showing LCZ classes to have distinct temperature profile using in situ air temperature measurement (Stewart et al., 2014) and simulations (Middel et al., 2014).

However, since air temperature data is generally in point form with geographically sparse measurement, a rather large literature use surface temperature (ST) to measure surface UHI (SUHI) as an indirect measure of UHI. Generally, satellite based thermal sensors are used (Peng et al., 2012), (Geletič et al., 2016) but UAV based methodologies (Sheng et al., 2010) has also been reported in literature. Among satellite based studies, Landsat $7 \& 8$ 's thermal products are popular due to higher spatial resolution, which aid in comparison between ST and urban form at finer level.

The initial objective of this study was similar; to measure SUHI in different built LCZ classes, as compared to green area in close vicinity. However, unlike existing literature which often only reports from specific date, the objective of this study was to see how SUHI varies across different seasons throughout the year. However, the study fails to find a definitive answer on SUHI characteristics of 
LCZ classes and reports a rather weak temperature difference between built and green LCZ. The study highlights the need for researchers to be careful in deploying Landsat thermal imagery, commonly taken during day time when the UHI intensity tends to be weak (Basara et al., 2008). Additionally, comments are drawn on comparison between remote sensing (RS) and GIS base LCZ classification and positive correlation between intensity of UHI and variance in ST of LCZ classes.

\section{Methodology}

While the Stewart and Oke (2012) framework envisage for a common methodology to achieve classification, data limitation and quality have resulted in divergent approaches. These methodologies can be broadly classified into remote sensing (RS) based and GIS based classification. Under former method, user defined Regions of Interests (ROIs), developed using high resolution imagery and local context knowledge, is used to train a classifier, which then generates LCZ map by classifying satellite imagery. On the other hand, for GIS based method, LCZ parameters developed by Stewart and Oke (2012) are calculated from vector data for pre-determined unit of analysis, and parameter ranges are used to determine each unit's LCZ class.

WUDAPT (See et al., 2015) (Bechtel et al., 2019), which aims to collect and curate LCZ classification identifies RS based method as Level 0 and eventually hope to integrate more vector data in higher levels. It is expected that such data would improve quality of classification. Thus initially in this research, LCZ classification using both methodologies is done to investigate if significant disagreement existing between two. To do so, firstly LCZ parameters were calculated for each census block, and those which satisfied all the parameters of at least one of LCZ class were identified (Map 1). These census blocks were converted to ROIs which are used to train Random Forest classifier, which is the algorithm of choice under WUDAPT. This classifier was then used to generate RS based LCZ map (Map 2) using Band 1-7, 10, 11 from Landsat 8 image. In parallel, a separate Random Forest classifier was trained using LCZ class parameter to estimate LCZ class of census block which did not fully satisfy conditions for any of LCZ classes (Map. 3).

The map $2 \& 3$ were filtered for the area occupied by building using map 4 as a mask. Since a Landsat image pixel might fall partially on building / non - building area, any pixel in which building cover exceeds $30 \%$ was also discarded. Then the two classifications was compared using an agreement matrix (Table 1). If a significant disagreement is found, the method in which standard deviation of ST for majority of LCZ class is lower, is selected since LCZ classification is based on unique thermal characteristic of each LCZ class (Bechtel et al., 2015). The ST used in the study are obtained from Landsat 8 provisional Surface Temperature available in Analysis Ready Data (ARD) (USGS, 2018) for North American region. In total, 46 instances of imagery were acquired between 03 Jan and 30 Dec for 2017. All the images are taken around 3:40 PM, local time. The mean interval was 7.7 days, with a maximum interval of 9 days. Some pixels $(<1 \%)$ were

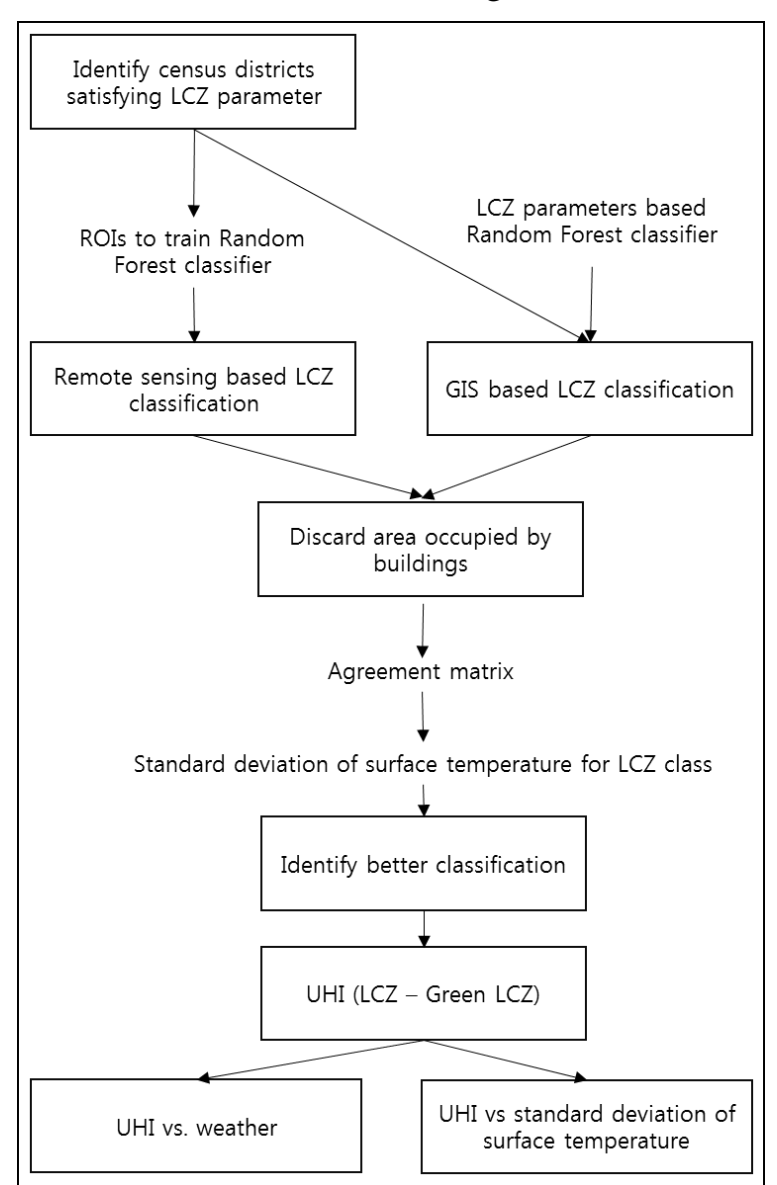

Figure 1. Summary of methodology 
discarded based on Surface Temperature Quality Assessment (STQA) band. Other than conversion from Kelvin to Celsius, no other processing was done.

Subsequently, UHI was calculated by subtracting mean ST of LCZ classes with mean ST of Green area (Fig. 4). It should be noted that due to data limitation, land use classes (A-G) were simplified as Green, Paved \& Water class system. Since the objective was to compare built LCZ classes (1-10) with green areas, this limitation did not have an effect on any of the analysis. As previously mentioned, we failed to see any major and consistent UHI effect. To explore causes behind inconsistent UHI intensity, the mean difference of ST for built classes was compared with general air temperature reported by weather station (Fig. 5) and with mean standard deviation of ST for built classes (Fig. 6).

Figure 1 provide a summary of above methodology.

\section{Results and Discussion}

As previously discussed, map 1 shows census blocks for which LCZ classes were identified using LCZ parameters developed by Stewart and Oke (2012). Map 2 and 3 shows RS and GIS based classification results. Figure 2 shows the area for each LCZ class from two classification methodology and table 1 compares agreement between two classifications (at remote sensing pixel level). Overall, GIS based classification identifies LCZ 3 and LCZ Paved quite less and LCZ 5 much more, as compared to RS based classification. If RS based classification is assumed to be 'true', GIS based classification grossly faltered in identifying LCZ 3 and Paved. On the other hand, if GIS based classification is assumed to be 'true', RS based classification faltered in identifying LCZ 4, 5, 6, 8, 10 , Water. Overall, the two classifications agreed on $52.89 \%$ of pixel's classes.

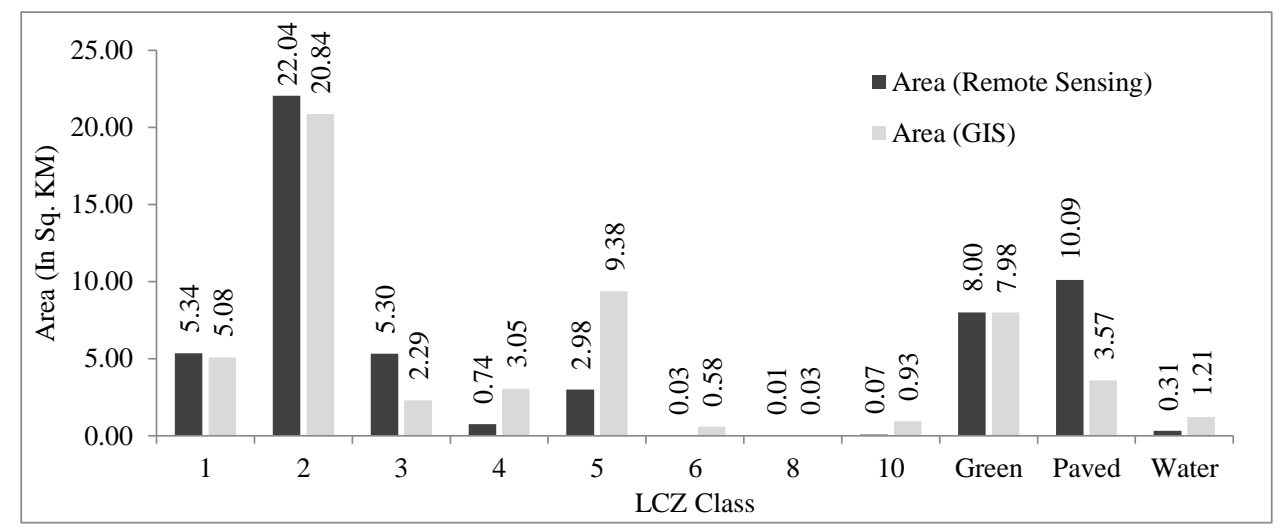

Figure 2. LCZ class area calculated by RS and GIS based classification

Table 1. LCZ class agreement between GIS and RS based classification.

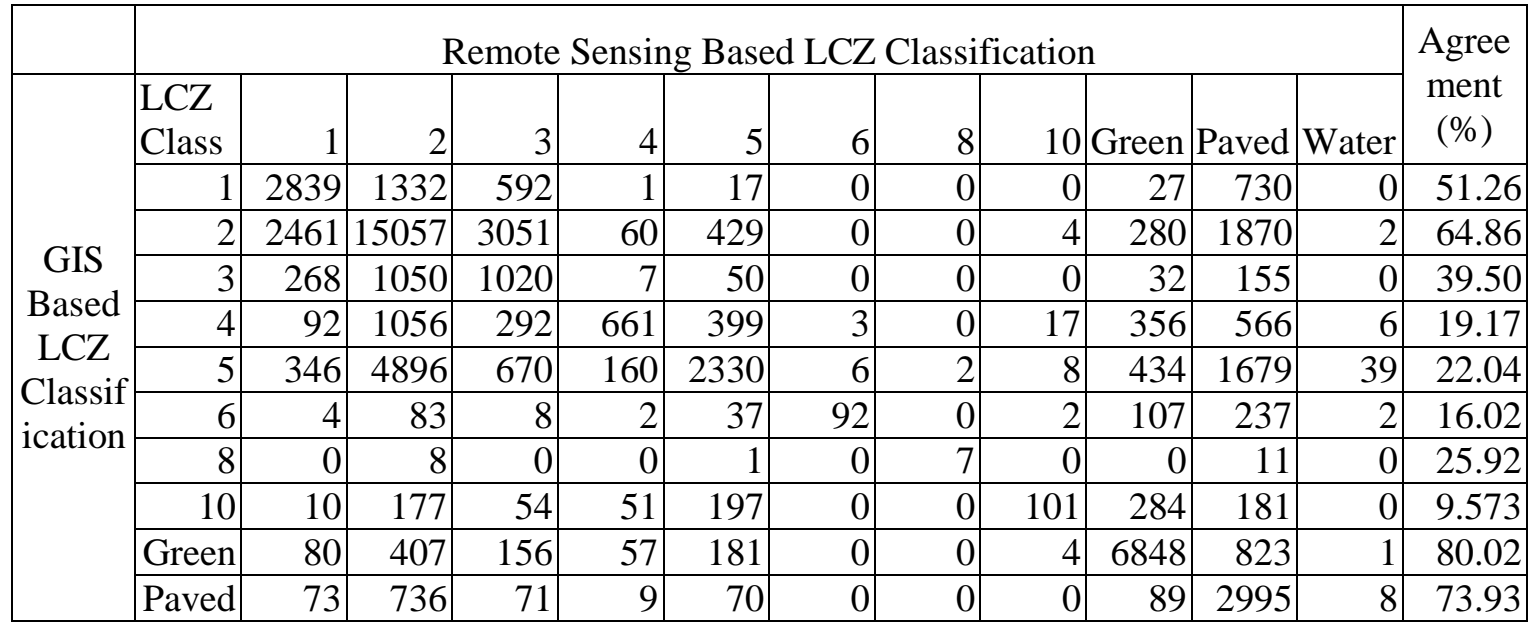




\begin{tabular}{|r|r|r|r|r|r|r|r|r|r|r|r|r|}
\hline Water & 6 & 35 & 1 & 0 & 19 & 0 & 0 & 0 & 575 & 406 & 292 & 21.88 \\
\hline Agreement(\%) & 45.94 & 60.62 & 17.24 & 65.57 & 62.46 & 91.08 & 77.77 & 74.26 & 75.81 & 19.37 & 83.42 & \\
\hline \multicolumn{10}{|c|}{ Overall Agreement $=52.89 \%$} \\
\hline
\end{tabular}

This is in contrast to literature such as (Gál et al., 2015) (Hidalgo et al., 2019), which have reported the RS and GIS based results to be fairly similar but agrees with findings of Hammerberg et al. (2018). The objective of study is not to determine cause of disagreement, but it can be speculated that disagreement stems from modifiable areal unit problem (MAUP). That is, while RS based classes were identified by $30 \times 30 \mathrm{M}$ pixel's properties, GIS based classes were identified based on aggregated census block's properties. To identify 'better' classification, standard deviation of ST for each class was calculated for each day (46 days) and summed up (Fig. 3). This is justifiable (to an extent) because better classification would produce classes which have minimum within class dissimilarity in terms of ST. From figure 3, it can be seen that RS based classification classes have similar or smaller standard deviation. Thus, for further investigations, RS based classification is used.

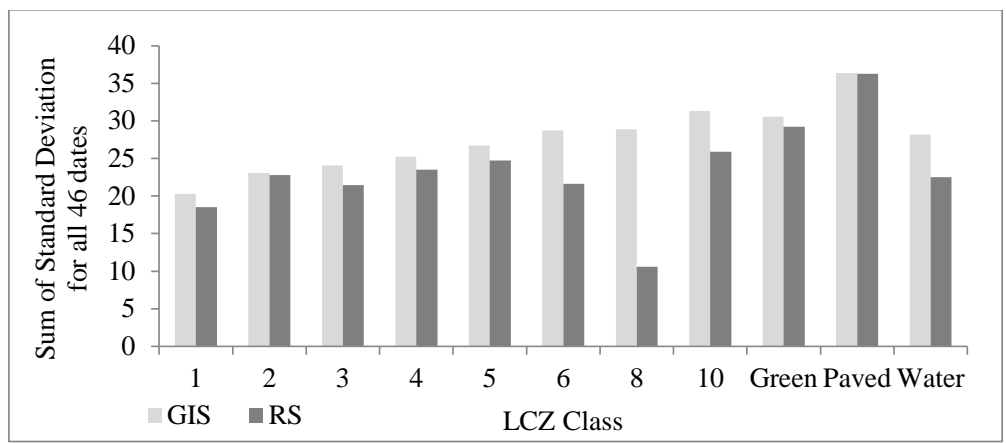

Figure 3. Comparison of surface temperature's standard deviation for RS and GIS based classification.

To identify which of LCZ classes (in which season) are prone to UHI, mean ST of the class was subtracted from mean ST of green areas (for the same date). Figure 4 shows the results. If \pm 0.5 is taken as a decision boundary for significant UHI (or cooling) the result indicate an ST difference to be immaterial except between May - Oct for most of LCZ classes. Even in this period, the difference in ST is not consistent, with seemingly random oscillation between relative hot and colds.

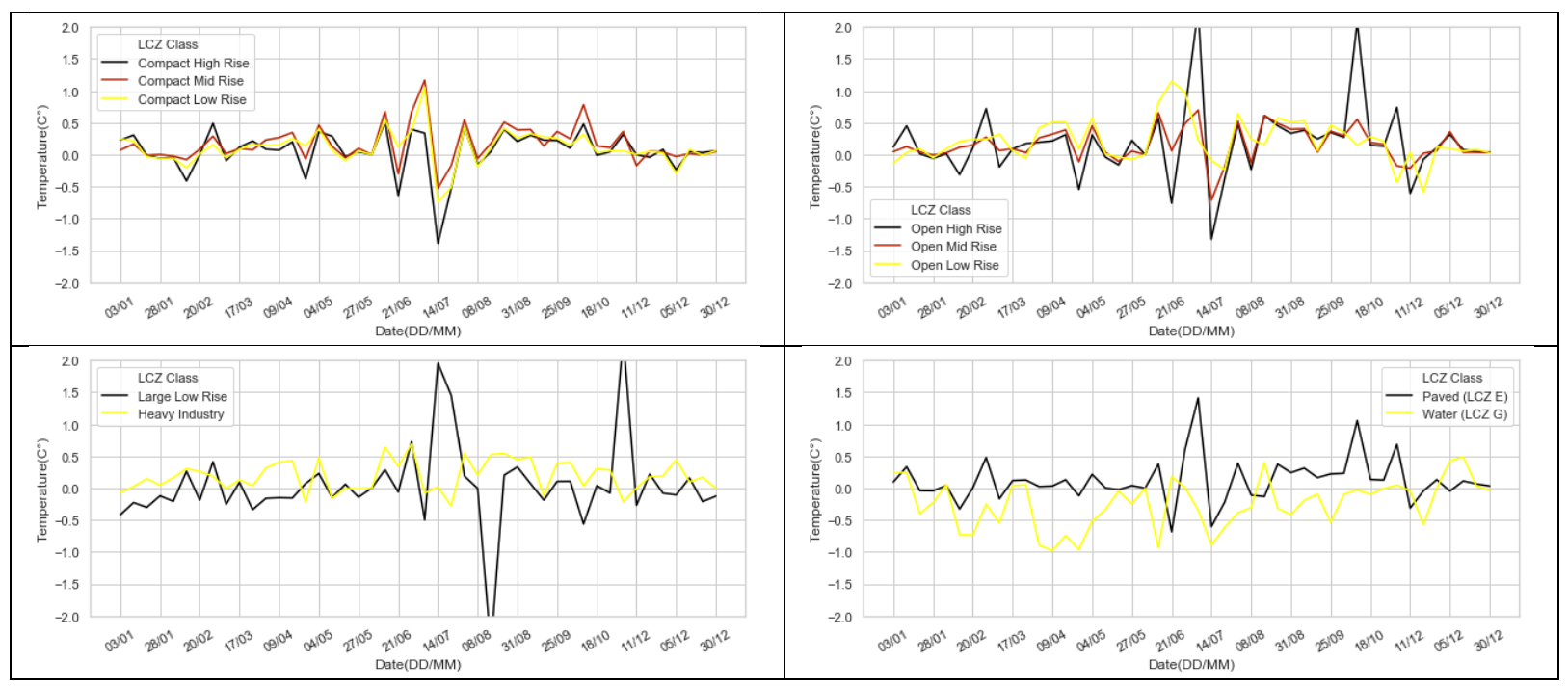

Figure 4. Difference between ST of LCZ classes and Green Area 
These results highlight the often ignored issue of using ST, especially daytime ST from different sensors including Landsat. As previously discussed, UHI is primarily observed after sunset with research suggesting a minor difference or even relativly cooler climate in urban area during the sunshine period. Since for Manhattan region Landsat 8 images are taken around 3:40 PM, the UHI effect seems to be minor for the majority of time period.

However, this does not explain why the magnitude of the fluctutation is different depending upon the time of year. To investigate, mean of ST difference for build LCZ class (LCZ 1-10) w.r.t green area is plotted against air temperature at 3:51 PM reported by ground weather station located in central park (Fig 5). Additionally, the mean standard deviation of ST for built LCZ class for respective date is shown in figure 6 .

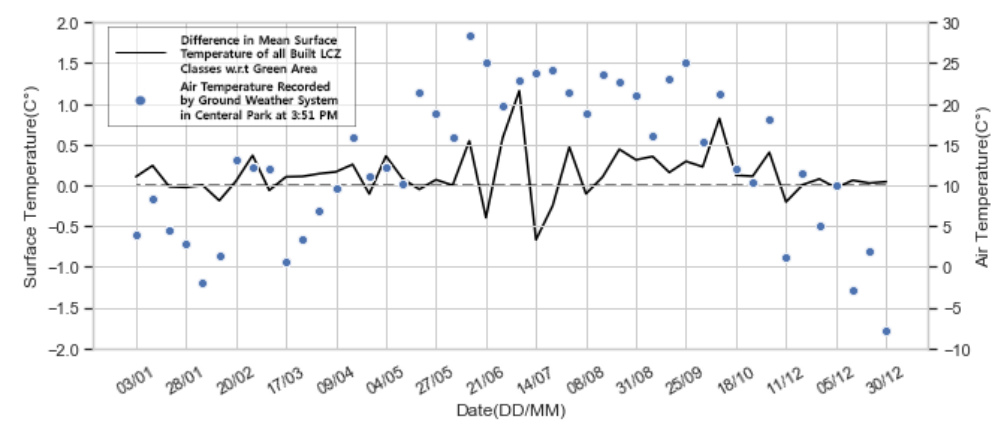

Figure 5. Difference in mean ST ( 3:40 PM) for built LCZ class w.r.t. green area vs. air temperature ( 3:51 PM) in central park.

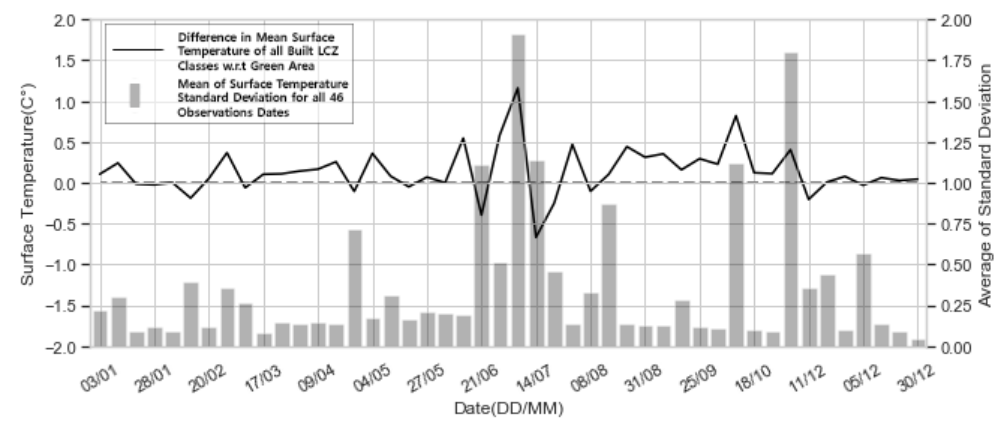

Figure 6. Difference in mean ST for built LCZ class w.r.t. green area vs. mean standard deviation in ST.

Figure $5 \& 6$ indicate that when the overall weather conditions are hotter, more fluctuation is observed. However at the same time, the spread of ST in a given class also tends to be higher. It is difficult to speculate the reasoning behind this and neither this is confirmation of such patterns to be spatially and temporally universal. Instead, the research points out need to further investigate the relation between general weather condition and the UHI in different LCZ classes.

\section{Conclusion}

In this research, negative results from quantifying UHI using ST from Landsat imagery in Manhattan are reported. For the majority of time period, the research failed to find significant differences in ST of built LCZ classes as compared to green area and when such differences were observed, the fluctuation in trend points toward major exogenous variable responsible for such observation. Thus, this work caution the researchers using ST, especially daytime ST for UHI investigations.

Additionally, the conflict between RS and GIS based LCZ classification is briefly discussed. The proposed framework for WUDAPT assumes GIS based classification to be superior to that based on RS, however, without due consideration for MAUP, this might not hold true. 


\section{References}

BASARA, J. B., HALL JR., P. K., SCHROEDER, A. J., ILLSTON, B. G. \& NEMUNAITIS, K. L. 2008. Diurnal cycle of the Oklahoma City urban heat island. Journal of Geophysical Research: Atmospheres, 113.

BECHTEL, B., ALEXANDER, P. J., BECK, C., BÖHNER, J., BROUSSE, O., CHING, J., DEMUZERE, M., FONTE, C., GÁL, T., HIDALGO, J., HOFFMANN, P., MIDDEL, A., MILLS, G., REN, C., SEE, L., SISMANIDIS, P., VERDONCK, M.-L., XU, G. \& XU, Y. 2019. Generating WUDAPT Level 0 data - Current status of production and evaluation. Urban Climate, 27, 24-45.

BECHTEL, B., ALEXANDER, P. J., BÖHNER, J., CHING, J., CONRAD, O., FEDDEMA, J., MILLS, G., SEE, L. \& STEWART, I. 2015. Mapping Local Climate Zones for a Worldwide Database of the Form and Function of Cities. 4, 199-219.

ESTRADA, F., BOTZEN, W. J. W. \& TOL, R. S. J. 2017. A global economic assessment of city policies to reduce climate change impacts. Nature Climate Change, 7, 403.

GÁL, T., BECHTEL, B. \& UNGER, J. 2015. Comparison of two different Local Climate Zone mapping methods. 9th International Conference on Urban Climate.

GELETIČ, J., LEHNERT, M. \& DOBROVOLNÝ, P. 2016. Land Surface Temperature Differences within Local Climate Zones, Based on Two Central European Cities. Remote Sensing, 8, 788.

HAMMERBERG, K., BROUSSE, O., MARTILLI, A. \& MAHDAVI, A. 2018. Implications of employing detailed urban canopy parameters for mesoscale climate modelling: a comparison between WUDAPT and GIS databases over Vienna, Austria. 38, e1241-e1257.

HIDALGO, J., DUMAS, G., MASSON, V., PETIT, G., BECHTEL, B., BOCHER, E., FOLEY, M., SCHOETTER, R. \& MILLS, G. 2019. Comparison between local climate zones maps derived from administrative datasets and satellite observations. Urban Climate, 27, 64-89.

MIDDEL, A., HÄB, K., BRAZEL, A. J., MARTIN, C. A. \& GUHATHAKURTA, S. 2014. Impact of urban form and design on mid-afternoon microclimate in Phoenix Local Climate Zones. Landscape and Urban Planning, 122, 16-28.

OKE, T. R. 2006. Towards better scientific communication in urban climate. Theoretical Applied Climatology, 84, 179-190.

PENG, S., PIAO, S., CIAIS, P., FRIEDLINGSTEIN, P., OTTLE, C., BRÉON, F.-M., NAN, H., ZHOU, L. \& MYNENI, R. B. 2012. Surface Urban Heat Island Across 419 Global Big Cities. Environmental Science \& Technology, 46, 696-703.

SEE, L., PERGER, C., DUeraUeR, M., FRITZ, S., BECHTEL, B., CHING, J., ALEXANDER, P., MiLlS, G., FOLEY, M., CONNOR, M. O., STEWART, I., FEDDEMA, J. \& MASSON, V. Developing a community-based worldwide urban morphology and materials database (WUDAPT) using remote sensing and crowdsourcing for improved urban climate modelling. 2015 Joint Urban Remote Sensing Event (JURSE), 30 March-1 April 2015 2015. 1-4.

SHENG, H., CHAO, H., COOPMANS, C., HAN, J., MCKEE, M. \& CHEN, Y. Low-cost UAV-based thermal infrared remote sensing: Platform, calibration and applications. Proceedings of 2010 IEEE/ASME International Conference on Mechatronic and Embedded Systems and Applications, 15-17 July 2010 2010. 38-43.

STEWART, I. D. \& OKE, T. R. 2012. Local Climate Zones for Urban Temperature Studies. 93, 18791900.

STEWART, I. D., OKE, T. R. \& KRAYENHOFF, E. S. 2014. Evaluation of the 'local climate zone' scheme using temperature observations and model simulations. International Journal of Climatology, 34, 1062-1080.

USGS 2018. U.S. Landsat Analysis Ready Data (Ard) Data Format Control Book. Version 5.0 ed. 


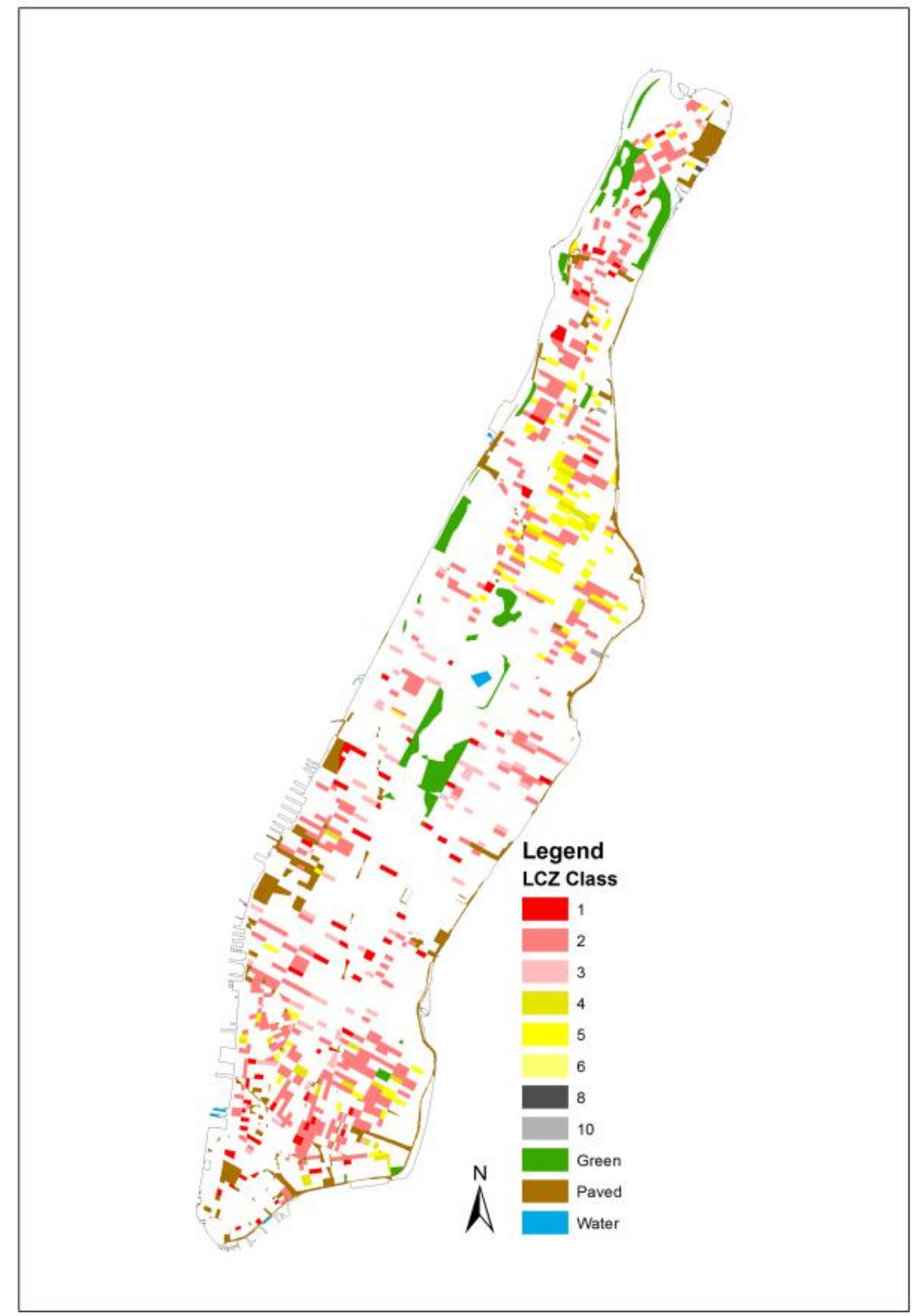

Map 1. Census blocks in Manhattan which satisfy all the parameters for at least one of LCZ Class. 


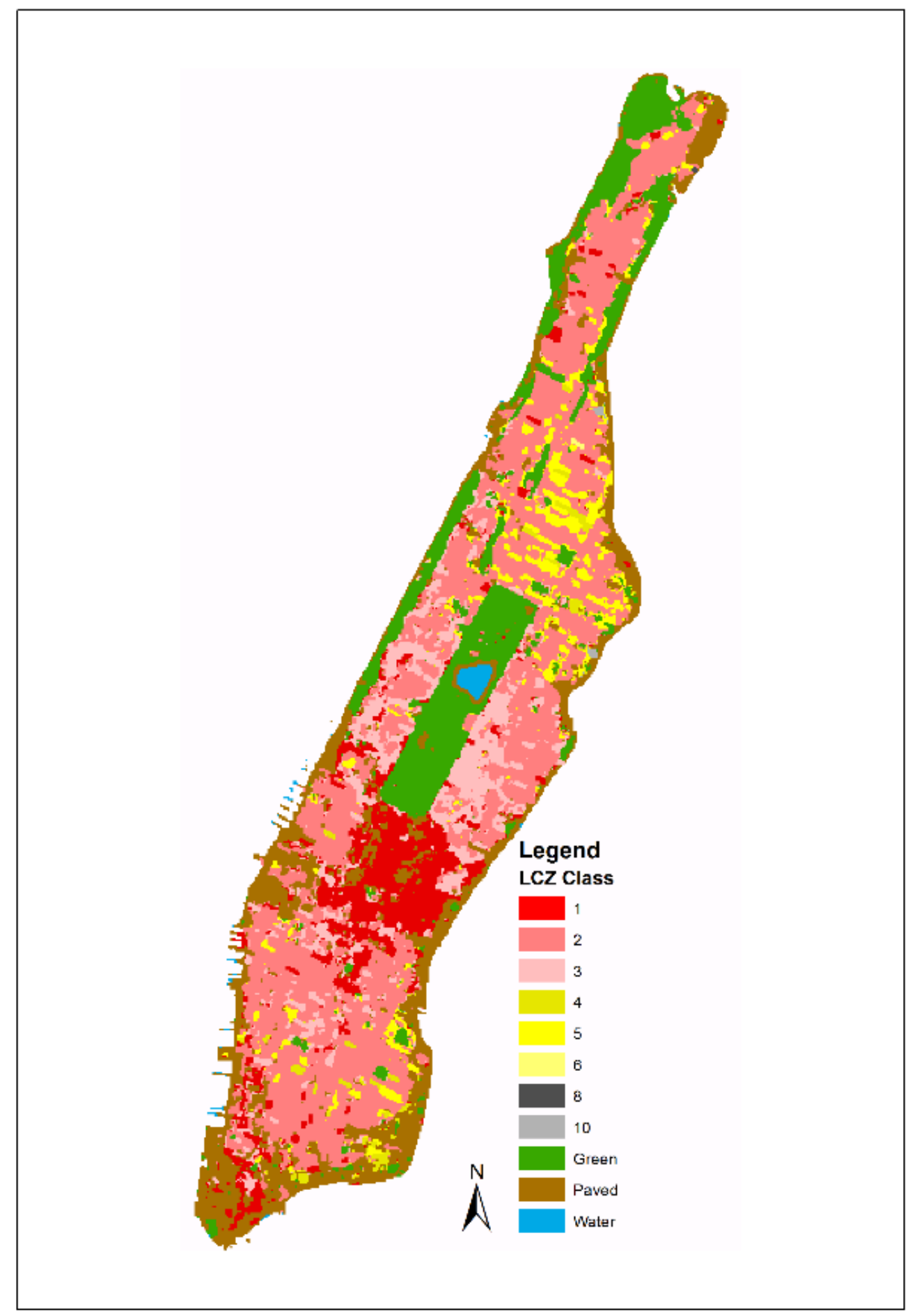

Map 2. Results of remote sensing based LCZ classification of Manhattan 


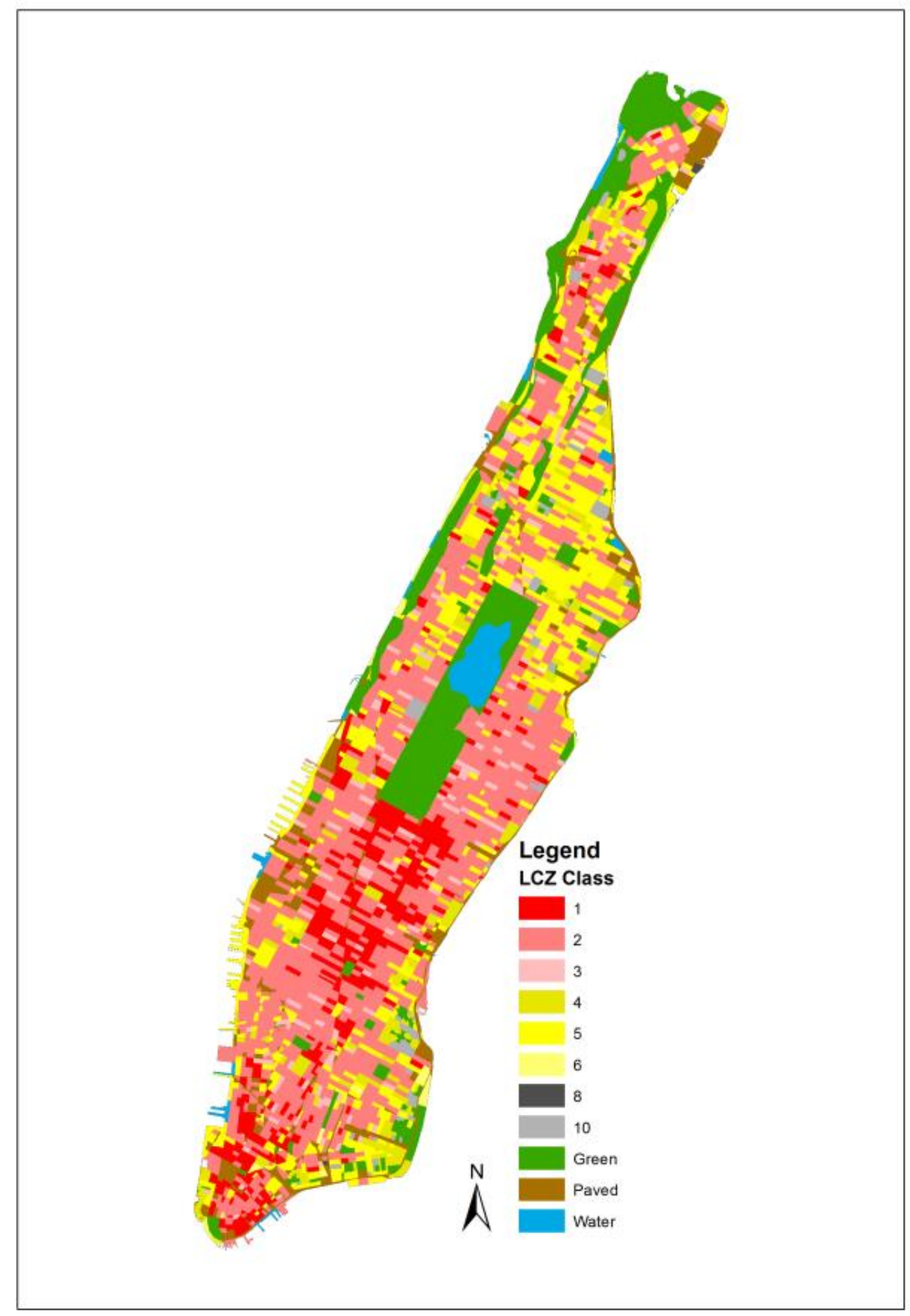

Map 3. Results of GIS based LCZ classification of Manhattan 


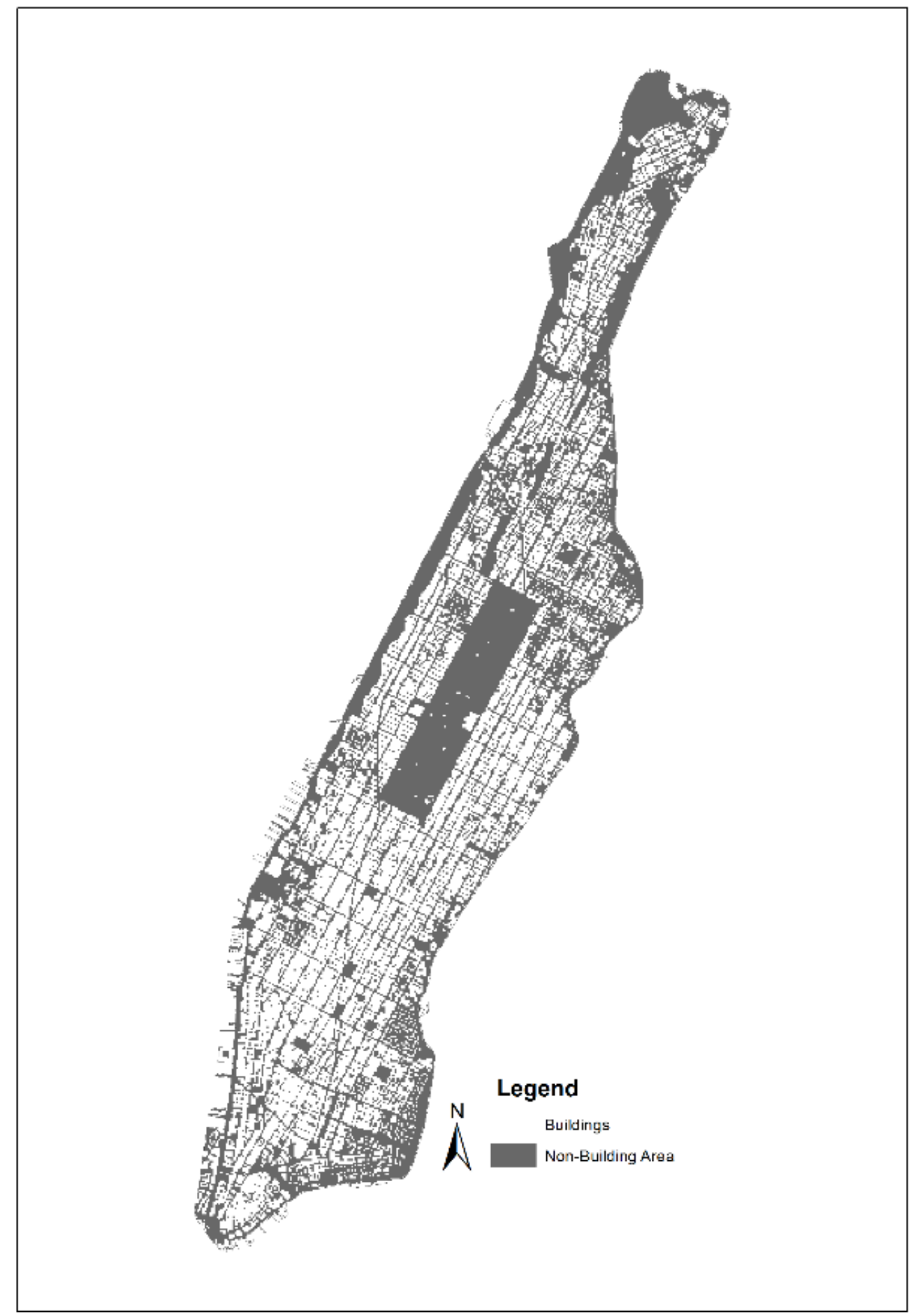

Map 4. Building vs non-building Area. Only surface temperature recoded at pixel with building cover less than $30 \%$ is used in this study. 